\title{
A CONTACT STRUCTURE FOR ODD DIMENSIONAL SPHERICAL SPACE FORMS
}

\author{
JOSEPH A. WOLF
}

Let $S^{2 n-1} \subset R^{2 n}$ denote an odd dimensional sphere of constant curvature $K>0$, embedded as the sphere of radius $K^{-1 / 2}$ in euclidean space. According to Gray [1] the linear differential form $\omega=\sum_{i=1}^{n}\left(x^{i} d x^{n+i}-x^{n+i} d x^{i}\right)$ defines a contact structure on $S^{2 n-1}$, i.e. the restriction satisfies $\omega \wedge(d \omega)^{n-1} \neq 0$. The unitary group $U(n)$ is embedded in the orthogonal group $O(2 n)$ as the set of all block form matrices

$$
\left(\begin{array}{rr}
A & B \\
-B & A
\end{array}\right)
$$

such that ${ }^{t} A \cdot A+{ }^{t} B \cdot B=I$ and ${ }^{t} A \cdot B={ }^{t} B \cdot A$. Now it is straightforward to check that $U(n)$ is the subgroup of all elements of $O(2 n)$ which preserve $\omega$. Let $\Gamma$ be a finite subgroup of $O(2 n)$ in which only the identity element has +1 for an eigenvalue. Then it follows from ([2], remarks on p. 155 , last column of chart on p. 208) that $\Gamma$ is conjugate in $O(2 n)$ to a subgroup of $U(n)$. On the other hand the $(2 n-1)$-dimensional complete connected riemannian manifolds of constant curvature $K>0$ are just the riemannian quotient manifolds $S^{2 n-1} / \Gamma$ with $\Gamma$ given as above. In summary, we have observed:

THEOREM. Let $M$ be a complete connected riemannian manifold of odd dimension $2 n-1$ and constant curvature $K>0$. Then $M$ inherits a contact structure from the linear differential form $\sum_{i=1}^{n}\left(x^{i} d x^{n+i}-x^{n+i} d x^{i}\right)$ on $S^{2 n-1}$.

\section{REFERENCES}

1. J. W. Gray, Some global properties of contact structures, Ann. of Math. 69 (1959), 421-450.

2. J. A. Wolf, Spaces of constant curvature, McGraw-Hill, New York, 1967.

University of California, Berkeley

Received by the editors November 28, 1966.

${ }^{1}$ Research partially supported by NSF Grant GP-5798. 